\title{
Puddle Dynamics and Air-to-Fuel Ratio Compensation for Gasoline-Ethanol Blends in Flex-Fuel Engines*
}

\author{
Kyung-ho Ahn, Anna G. Stefanopoulou, and Mrdjan Jankovic
}

\begin{abstract}
Ethanol is being increasingly used as an alternative fuel to petroleum-based gasoline and diesel derivatives. Currently available flexible fuel vehicles (FFVs) can operate on a blend of gasoline and ethanol in any concentration of up to $85 \%$ ethanol (93\% in Brazil) with minimum hardware modifications. This flexibility is partly achieved through the closed loop airto-fuel ratio control which maintains automatically operation around the stoichiometric ratio. Precise transient AFR control depends however on a feedforward compensator that reduces the transient effects of fuel puddle dynamics. An accurate and tunable model of the fuel puddle dynamics for gasoline-ethanol blends is, thus, necessary for the purpose of air-to-fuel ratio control. In this paper, we propose a physics-based fuel puddle model that may be used for control purposes in FFVs. In particular, the gasoline-ethanol blend is modeled using several chemical compounds and is parameterized by ethanol content. The model consists of a droplet evaporation model and a singlepuddle vaporization model. The droplet evaporation model is simulated off-line to generate port wall-impacting factors of injected fuel to be used in a single-puddle vaporization model. The single-puddle vaporization model is a cycle-based model that may be simulated on-line to characterize fuel puddle dynamics in port fuel injected engines. To verify the validity of the model, simulation results are compared with limited experimental data. A transient fuel compensator based on the proposed model is also formulated.
\end{abstract}

Index Terms-Fuel dynamics, multi-component fuel, evaporation, air-to-fuel ratio control, gasoline-ethanol blend, flex-fuel vehicles

\section{NOMENCLATURE}

$A F R \quad$ Air-to-fuel ratio.

$B \quad$ Transfer number or Spalding number.

$e \quad$ Volume fraction of ethanol in a fuel blend.

ECT Engine coolant temperature (K).

$e_{m} \quad$ Mass fraction of ethanol in a fuel blend.

$D \quad$ Diffusion coefficient $\left(\mathrm{m}^{2} / \mathrm{s}\right)$.

$d_{d} \quad$ Droplet diameter $(\mathrm{m})$.

$d_{\text {in }} \quad$ Port diameter $(\mathrm{m})$.

$f \quad$ Mass fraction of a fuel component.

$M \quad$ Molecular weight $(\mathrm{kg} / \mathrm{kmol})$.

$m_{\text {air }} \quad$ Cylinder air charge $(\mathrm{kg})$.

$m_{i n j} \quad$ Injected fuel mass $(\mathrm{kg})$.

K. Ahn and A. Stefanopoulou are with the Department of Mechanical Engineering, University of Michigan, Ann Arbor, MI 48109-2121 USA (email: akyungho@umich.edu; annastef@umich.edu).

M. Jankovic is with the Ford Research and Advanced Engineering, Dearborn, MI 48121 USA (e-mail: mjankov1@ ford.com).

*Funds provided from a UMICH/Ford Alliance grant.

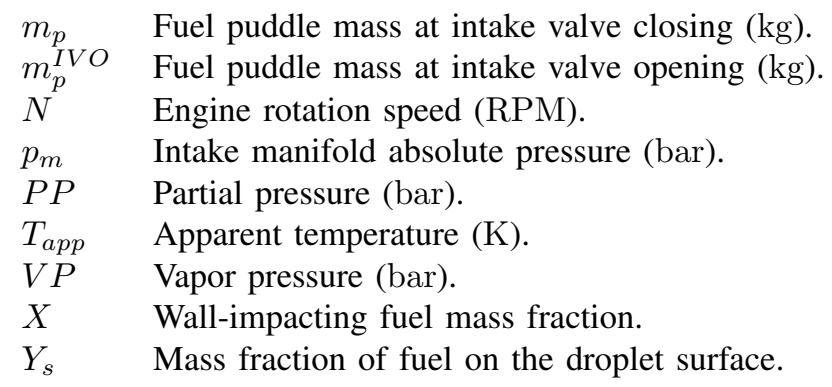

Greek:

$\delta_{t h} \quad$ Fuel film thickness (m).

$\lambda \quad$ Relative air-to-fuel ratio.

$\rho \quad$ Fuel density $\left(\mathrm{kg} / \mathrm{m}^{3}\right)$.

$\tau_{D A} \quad$ Time constant for number decay (s).

\section{INTRODUCTION}

C URRENTLY available flexible fuel vehicles (FFVs) can operate on a blend of gasoline and ethanol in any concentration of up to $85 \%$ ethanol. The blend is denoted by the EXX nomenclature, where XX represents the volumetric percentage of ethanol in the blend. The United States commonly uses E85 as an alternative to the normal E0 or gasoline fuel. In Brazil, however, the fuel blend also contains water and E100 refers to a blend of 93\% ethanol and 7\% of water [6]. Such fuel blends mixed with the addition of water are not considered in this paper. Given the effect of fuel variation, FFVs should embed engine calibration maps in their controllers and management systems to account for this variation. One of such calibration map would account for the effect of ethanol concentration on the fuel puddle dynamics and the associated fuel injection compensation for tight air-tofuel regulation of the stoichiometric ratio of each fuel.

Air-to-fuel ratio control around the stoichiometric ratio of a fuel blend is important to meet stringent emission requirements for spark ignition (SI) engines. For a given air charge, the stoichiometric fuel is typically achieved by a combination of feedforward and feedback control on the fuel injection. The feedback controller is based on the measured ratio $(\lambda)$ of the actual air-to-fuel ratio $(A F R)$ to the stoichiometric ratio $\left(A F R_{s}\right)$ through an exhaust gas oxygen sensor. The $\lambda$ ratio is compared to $\lambda^{\text {des }}=1$ and the error is used by a proportional-integral (PI) controller to adjust the fuel command. Due to the long delays in the feedback loop, most engine controllers employ a feedforward fuel command which 
TABLE I

MODEL FUEL FOR GASOLINE

\begin{tabular}{|c|c|c|c|c|c|c|c|}
\hline $\begin{array}{l}\text { Component/ } \\
\text { Mass fraction }\end{array}$ & $\begin{array}{c}\text { Molecular } \\
\text { Formula }\end{array}$ & $\begin{array}{c}\text { Molecular } \\
\text { Weight }\end{array}$ & $\begin{array}{c}\text { Normal Boiling } \\
\text { Point }(\mathrm{K})\end{array}$ & SAE 982519 [5] & SAE 982724 [7] & SAE 2005-01-1127 [2] & This Paper \\
\hline n-butane & $\mathrm{C}_{4} \mathrm{H}_{10}$ & 58.123 & 272.7 & - & - & 0.04 & - \\
\hline iso-pentane & $\mathrm{C}_{5} \mathrm{H}_{12}$ & 72.151 & 301.0 & 0.1618 & 0.1330 & 0.16 & - \\
\hline cyclo-hexane & $\mathrm{C}_{6} \mathrm{H}_{12}$ & 84.162 & 353.8 & 0.2091 & 0.1893 & - & 0.65 \\
\hline n-hexane & $\mathrm{C}_{6} \mathrm{H}_{14}$ & 86.177 & 341.9 & - & - & 0.025 & - \\
\hline iso-octane & $\mathrm{C}_{8} \mathrm{H}_{18}$ & 114.232 & 372.4 & 0.1670 & 0.1493 & 0.32 & - \\
\hline toluene & $\mathrm{C}_{7} \mathrm{H}_{8}$ & 92.141 & 383.8 & 0.1535 & 0.1824 & 0.255 & - \\
\hline ethyl-benzene & $\mathrm{C}_{8} \mathrm{H}_{10}$ & 106.168 & 409.3 & 0.1559 & 0.1462 & - & 0.20 \\
\hline 123 trimethyl-benzene & $\mathrm{C}_{9} \mathrm{H}_{12}$ & 120.194 & 449.3 & - & - & 0.18 & - \\
\hline n-decane & $\mathrm{C}_{10} \mathrm{H}_{22}$ & 142.286 & 447.3 & 0.1171 & 0.1210 & - & 0.11 \\
\hline naphthalene & $\mathrm{C}_{10} \mathrm{H}_{8}$ & 128.174 & 491.1 & 0.0355 & 0.0319 & - & 0.04 \\
\hline n-tridecane & $\mathrm{C}_{13} \mathrm{H}_{28}$ & 184.365 & 508.6 & - & - & 0.02 & - \\
\hline ethanol & $\mathrm{C}_{2} \mathrm{H}_{6} \mathrm{O}$ & 46.069 & 351.4 & - & 0.0469 & - & - \\
\hline
\end{tabular}

is primarily derived from the estimated cylinder air charge divided by the assumed stoichiometric ratio of the assumed fuel blend. Furthermore, the feedforward is usually designed to eliminate the transient effects of fuel puddle dynamics in port fuel injected (PFI) engines.

Since the puddle dynamics and hence the fuel entering the cylinder depend on the ethanol content, transient fuel compensation (TFC) should be based on the gasoline-ethanol content and should retain the ability to be used in wide range of operating conditions. To this end, it is desirable to design a low-order model with a few tunable physical parameters.

A model for transient fuel compensation of gasoline in the form of a simple linear time invariant model for the fuel puddle dynamics was first introduced by Aquino in the early 1980s [1]. Curtis et al. developed the four puddle model (FPM) in which fuel films are distributed in four different locations in a PFI engine and the physics of fuel vaporization is well-exploited with use of multi-component fuel model [4]. Locatelli et al. in [13] introduced a simple control-oriented wall-wetting model based on physical principles, where the port injected fuel dynamics is modeled as uniform droplet vaporization and exponential decay of the number of airborne droplets. An extended Kalman filter is used then to identify the physical parameters of the puddle dynamics for a gasoline fuel in [14]. The proposed model in this paper has the same basic structure as that of the model by Locatelli et al. [13] but extended to account for the vaporization of the various primitive chemical components that comprise the gasolineethanol blends. Adapting [13] for an aggregate gasolineethanol fuel blend by interpolation between the gasoline and ethanol volatility curves was found inadequate for two reasons. First, the volatility curves obtained at standard atmospheric pressure do not account for the strong vaporization dependency on manifold pressure [17]. Second, the volatility curve of E85 is very steep at the boiling temperature of ethanol. There should be then huge uncertainty in obtaining the vaporized mass fraction in the model at liquid fuel temperature near the boiling temperature. Almost no vaporization is obtained below the boiling temperature and almost all vaporization is obtained above the boiling temperature. The second inadequacy actually arises from misinterpreting the fuel volatility experiments used primarily for the classification of fuels [7]. Fuel volatility curves are obtained by the distillation process in which a volume of liquid fuel whose exposed surface area is relatively small is boiled by applying heat to the liquid. However, in the injected fuel spray and the fuel film on the intake wall, not only the total volume of liquid fuel is small but also the ratio of volume to the exposed surface area is small, thus causing more chance of evaporation before the liquid temperature reaches the boiling temperature. In addition, the volatility curve cannot be utilized to evaluate certain property instantaneously since the vaporized fraction obtained by the volatility curve is a cumulative sum over a certain heating period.

Batteh and Curtis introduced a fuel puddle dynamic model with alternative fuels in 2005 [2]. They used the four puddle model but unfortunately the fuel was modeled as a different combination of several fuel components for each fuel blend, i.e. indolene, E22 and E85 not allowing a continuous variation within the E0-E85 range. Their model is actually not an invertible model, which was not fundamentally developed for transient compensator design.

In this paper, we present a physics-based single puddle model using multi-component model fuel for FFVs. The model is divided into two parts: droplet evaporation model to generate the wall-impacting portion of the injected fuel and the single puddle vaporization model ${ }^{1}$. To capture the characteristics of the physical vaporization process, a multi-component fuel model parameterized by the ethanol content is utilized. To verify the validity of the model, model simulation results are compared with the experimental data provided in [2] for E0, E22 and E85. A transient fuel compensator using the proposed model is also formulated. Since direct pole-zero cancelation similar to the $X$ and $\tau$ pre-compensator of [15] is not possible for the nonlinear multi-component fuel puddle model, where $X$ and $\tau$ are the wall impacting factor and the fuel film

\footnotetext{
${ }^{1}$ This structure is the same as that of the model by Locatelli et al [13].
} 
vaporization time constant, respectively, introduced in [1], an approximation is used to invert the model to design a TFC. The approximation is demonstrated with a TFC simulation.

\section{Study with a Simple Fuel Puddle Model for A SPECIFIC FUEL BLEND}

In this section, we introduce a simple fuel puddle model for a specific fuel blend with a fixed volume fraction of ethanol, $e$, in the fuel blend based on the puddle model introduced in [11] which is adapted from Aquino's $X-\tau$ model [1]. The purpose of this section is to demonstrate the nonlinear variation of model parameters with change of the ethanol content, which are identified from experimental data, and hence to justify the need for the proposed model. The parameter calibration of $X$ and $\tau$ is not a simple procedure in general because these two parameters depend on many engine variables such as pressure, flow and temperature [11], [3], [16]. Adding fuel composition variability increases further the complexity of the parameterization. Moreover, we show below that parameterizing the puddle dynamics using the Aquino's model structure for the two extreme ethanol concentrations, namely E0 and E85, does not allow interpolation for capturing the puddle behavior for intermediate ethanol blends.

The mass of the fuel puddle at intake valve opening, $m_{p}^{I V O}$, is equal to the sum of the previous-cycle fuel puddle mass, $m_{p}$, and the fraction of the newly injected fuel $m_{i n j}$ that hits the puddle. The mass of fuel at intake valve closing, $m_{p}$, is reduced by the amount of evaporated fuel during the intake stroke, $m_{\text {evap }}$ :

$$
\begin{aligned}
m_{p}(k) & =m_{p}(k-1)+X(k) m_{\text {inj }}(k)-m_{\text {evap }}(k), \\
m_{f, \text { cyl }}(k) & =(1-X(k)) m_{\text {inj }}(k)+m_{\text {evap }}(k),
\end{aligned}
$$

where $m_{f, c y l}(k)$ is the fuel mass inducted into the cylinder at step $k, X$ is a time-varying parameter which is the fraction of injected fuel that hits the puddle and $m_{p}^{I V O}(k)$ is expressed by:

$$
m_{p}^{I V O}(k)=m_{p}(k-1)+X(k) m_{i n j}(k) .
$$

This model, (1) and (2), has exactly the same structure as introduced in [11]. To simplify the evaporation model, we introduce another time-varying parameter $a$ in place of $\tau$ so that the evaporated mass at step $k$ is expressed as $m_{\text {evap }}(k)=a(k) m_{p}^{I V O}(k)$. The parameter $a$ is the evaporated mass fraction during the intake valve open from the puddle mass at intake valve opening. The fuel puddle model is then expressed as:

$$
\begin{aligned}
& m_{p}^{I V O}(k)=(1-a(k-1)) m_{p}^{I V O}(k-1)+X(k) m_{i n j}(k), \\
& m_{f, c y l}(k)=(1-X(k)) m_{i n j}(k)+a(k) m_{p}^{I V O}(k) .
\end{aligned}
$$

For parameter identification using the experimental data for each fuel blend, we include $m_{i n j}$ and $m_{f, c y l}$ in our observations since $m_{i n j}$ can be obtained by the commanded injection amount and $m_{f, c y l}$ can be calculated by using the measured (and approximated) cylinder air charge, measured UEGO data $(\lambda)$ and appropriate transport delay and a time lag associated with the engine/sensor process. Let us define the following two variables for convenience:

$$
\bar{a}(k) \triangleq 1-a(k), \quad \bar{X}(k) \triangleq 1-X(k) .
$$

From (5), $m_{p}^{I V O}(k)$ is expressed as:

$$
m_{p}^{I V O}(k)=\frac{1}{a(k)}\left(m_{f, c y l}(k)-\bar{X}(k) m_{i n j}(k)\right) .
$$

By eliminating $m_{p}^{I V O}$ in (4) using (7), we obtain:

$$
\begin{aligned}
& m_{f, c y l}(k)-m_{i n j}(k)= \\
& \frac{a(k)}{a(k-1)} \bar{a}(k-1)\left(m_{f, c y l}(k-1)-\bar{X}(k-1) m_{i n j}(k-1)\right) \\
& -\bar{a}(k) X(k) m_{i n j}(k) .
\end{aligned}
$$

Assume that $a$ is approximately the same between two consecutive cycles. By applying $a(k) / a(k-1)=1$, the above equation is simplified to:

$$
\begin{aligned}
& m_{f, c y l}(k)-m_{i n j}(k)=\bar{a}(k-1) m_{f, c y l}(k-1) \\
& -\bar{a}(k-1) \bar{X}(k-1) m_{i n j}(k-1)-\bar{a}(k) X(k) m_{i n j}(k) .
\end{aligned}
$$

By introducing $\bar{b}(k) \triangleq \bar{a}(k) \bar{X}(k)$, (8) is expressed in a convenient form for regression:

$$
\begin{aligned}
& m_{f, c y l}(k)-m_{i n j}(k)=m_{f, c y l}(k-1) \bar{a}(k-1) \\
& -m_{i n j}(k) \bar{a}(k)-m_{i n j}(k-1) \bar{b}(k-1)+m_{i n j}(k) \bar{b}(k) .
\end{aligned}
$$

We used each experimental data set of E0, E22 and E85 as shown in Fig. 6 and Fig. 8 for E0 fuel case, for parameter identification of $\bar{a}$ and $\bar{b}$ and hence $X$ and $a$ for each fuel blend. For the limited test data available, one engine speed and two manifold pressures and associated air and fuel flows during the warm-up phase for three fuels were available. The parameters $X$ and $a$ were, hence, only modeled as functions of engine coolant temperature. Parameters $\bar{a}$ and $\bar{b}$ were actually regressed as second order polynomials of engine coolant temperature, $E C T$, with coefficients identified by standard least squares technique. Data from the very early phase of the engine start period were eliminated since engine RPM steeply increases. Half of the remaining data was utilized for the least squares fit and the other half was used for validation involving comparison of the experimental data and the identified model output. Fig. 1 depicts the air-to-fuel ratio comparison between

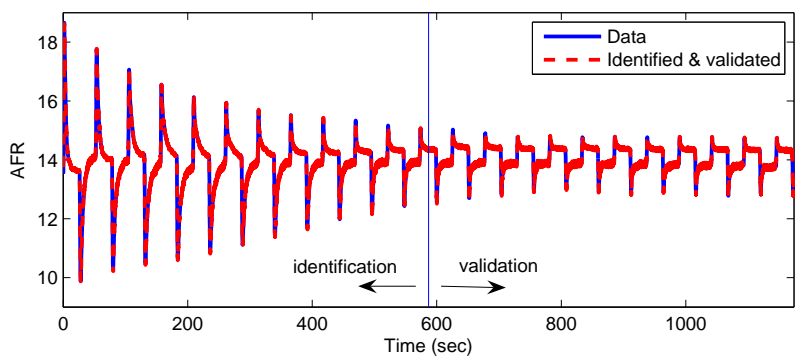

Fig. 1. Air-to-fuel ratio comparison between the experimental data and the identified model output for E0 

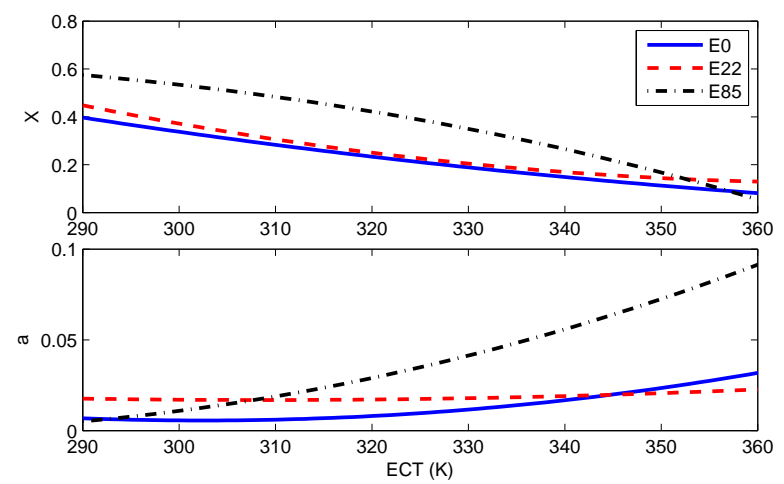

Fig. 2. Identified fuel puddle model parameters for E0, E22 and E85

the data and the model output for the data set of E0, showing good parameter fitting result. Fig. 2 shows the identified fuel puddle model parameters for each fuel blend versus the engine coolant temperature. It is observed that the parameters for E0, E22 and E85 are different and neither parameter for E22 can be uniformly interpolated between the E0 and E85 parameters. Based on this observation, it is not clear how many fuel blends one needs to test in order to identify and adequately interpolate the puddle parameters in addition to the fuel specific parameterizations for engine coolant temperature, manifold absolute pressure and engine RPM [11]. Note that on-line identification of the $X$ and $a$ parameters [20] is very challenging due to the fast changes in the parameters space (RPM, MAP, ECT) during typical driving scenarios. Therefore, even though fuel puddle dynamics seems to be a fairly simple dynamic process, a certain level of complexity involved with a physics-based model may be inevitable to appropriately capture the different dynamic characteristics for different fuel blends.

\section{Multi-Component Model Fuel}

The multi-component model fuel for gasoline can be constructed by selecting a combination of several fuel compounds which mimics the volatility of real fuel as in [7]. Table I shows such specific combinations for gasoline model listed in [5], [7] and [2] and the combination used in this paper. The mass fractions used in this paper were determined by reducing fuel components and adjusting mass fractions from the combination of mass fractions of [5] until the error between the modeled and measured [2] $\lambda$ converged to a small value after the model tuning constants were fixed to match the experimental data for the model fuel of [5]. The experimental data targeted to provide the model tuning are the $\lambda$ responses from the engine warm-up tests with several tip-ins and tip-outs as described in [2] and shown in section VI where simulations are discussed.

A gasoline-ethanol blend is then modeled as a combination of 5 chemical compounds including ethanol. Let $e$ and $e_{m}$ denote the volume fraction and the mass fraction of ethanol in a gasoline-ethanol blend, respectively. The mass fraction $e_{m}$ is calculated by:

$$
e_{m}=\frac{e}{e+(1-e) / 1.056} .
$$

Let $f_{g, i}$ denote the mass fraction of each component for the gasoline model fuel where $i=1, \cdots, 4$ is the index representing each component, which is directly read from the last column in Table I. The mass fraction of each component in the fuel blend is then expressed as:

$$
\begin{aligned}
f_{i} & =f_{g, i} \times\left(1-e_{m}\right), \quad i=1, \cdots, 4, \\
f_{5} & =e_{m} .
\end{aligned}
$$

\section{Droplet Evaporation Model}

It is assumed that spherical shape droplets of the same size are formed right after fuel injection. The total number of airborne droplets are modeled as an exponential decaying process [13]. The number of droplets is reduced by hitting the port wall. Droplets vaporize while they are airborne. The total mass of vaporization from droplets is regarded as the fuel mass inducted into the cylinder. A mathematical model for this process is constructed as follows. All fuel is injected as droplets of initial diameter $d_{d}^{0}$ with resulting initial droplet mass $m_{d}^{0}=\frac{\pi}{6} \rho^{0}\left(d_{d}^{0}\right)^{3}$ and initial mass of each component in a droplet $m_{d, i}^{0}=f_{i} \cdot m_{d}^{0}$, where $\rho^{0}$ denotes the initial density of mixture. Each droplet is then reduced by the droplet evaporation. Assuming stagnant air surrounding a droplet ${ }^{2}$ and fuel vapors far from the droplet surface being zero, the evolution of each droplet mass $m_{d}$ and its individual component masses $m_{d, i}$ are dictated by the droplet evaporation rate $\dot{m}_{E V, d}$ as in [18], [19], [25]:

$$
\begin{aligned}
\frac{d}{d t} m_{d} & =-\dot{m}_{E V, d}, \\
\frac{d}{d t} m_{d, i} & =-Y_{s, i} \cdot \frac{1+B_{d}}{B_{d}} \cdot \dot{m}_{E V, d}
\end{aligned}
$$

with

$$
\dot{m}_{E V, d}=2 \pi \cdot d_{d} \cdot \rho \cdot D \cdot \ln \left(B_{d}+1\right)
$$

where the droplet diameter follows the droplet mass reduction $d_{d}=\left(\frac{6 m_{d}}{\pi \rho}\right)^{1 / 3}, Y_{s, i}$ denotes the mass fraction of each fuel component above the droplet, $B_{d}$ is the transfer number, $D$ is diffusion coefficient of the fuel vapor, and the specific volume $1 / \rho$ is calculated as mass-weighted average of specific volumes of each fuel component. Although the experimentally observed vapor pressure for gasoline-ethanol blends deviates from the one expected from ideal mixtures as shown in [12], we neglect this complexity and use Raoult's law to our controloriented model. According to Raoult's law [23], the mass fraction of each fuel component above the droplet is expressed as:

$$
Y_{s, i}=\frac{V P_{i} \times f_{d, i}}{\sum_{i}\left(V P_{i} \times f_{d, i}\right)+P P_{a i r} \cdot M_{a i r} \cdot \sum_{i} f_{d, i} / M_{i}} .
$$

The transfer number $B_{d}$ is calculated as follows [2], [21], [23]:

$$
B_{d}=\frac{\sum_{i} V P_{i} \times f_{d, i}}{P P_{a i r} \times M_{a i r} \times \sum_{i} f_{d, i} / M_{i}},
$$

${ }^{2}$ Although restrictive, this assumption is reasonable because fuel is injected before the intake stroke, hence, before any significant air flow surrounds the droplets. 


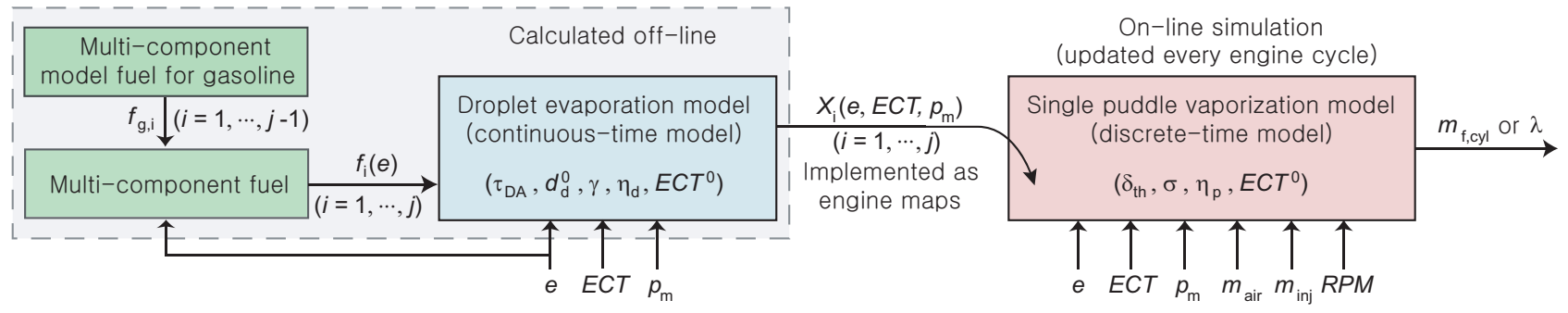

Fig. 3. Schematic of fuel puddle dynamic model for gasoline-ethanol E0-E85 blend

with the partial pressure of air calculated from

$$
P P_{\text {air }}=p_{m}-\frac{\sum_{i} V P_{i} \times f_{d, i} / M_{i}}{\sum_{i} f_{d, i} / M_{i}},
$$

where summation $\sum_{i}$ is performed over all fuel components, $V P_{i}$ denotes the normal vapor pressure of the $i$-th fuel component, $M_{i}$ is the molecular weight of the $i$-th fuel component, $M_{a i r}$ is the molecular weight of air $(28.97 \mathrm{~g} / \mathrm{mol}), p_{m}$ denotes the manifold absolute pressure (MAP) and $f_{d, i}$ is defined as the mass fraction of the $i$-th fuel component in the droplet:

$$
f_{d, i} \triangleq \frac{m_{d, i}}{\sum_{i} m_{d, i}}=\frac{m_{d, i}}{m_{d}} .
$$

The diffusion coefficient, $D$, and the normal vapor pressures, $V P_{i}$, should be evaluated at the droplet surface temperature. However, it is hard to exactly predict the temperature. Instead, we use an apparent temperature which is parameterized by the ethanol content, $e$, and the engine coolant temperature, $E C T$ :

$$
T_{a p p, d}=E C T-(e+\gamma) \cdot \eta_{d} \cdot\left(E C T-E C T^{0}\right),
$$

where $\gamma, \eta_{d}$ and $E C T^{0}$ are three tuning constants. There are publications of fuel puddle dynamic models which include temperature dynamics of explicit states, i.e. differential equations for some temperatures, e.g. valve, cylinder and port temperatures [2], [13]. The purpose of a dynamic temperature model is to explicitly evaluate a physical property at the temperature of a designated location. However, it is still difficult to establish a simple dynamic model to predict the droplet surface temperature and a dynamic temperature model usually needs several tuning constants in the equations. A dynamic temperature model may be introduced to our model in future study. Nevertheless, in this paper, we regard a temperature change approximately as a static process parameterized by the engine coolant temperature, ECT, which is measured on-line. The apparent temperature does not play a role of approximating a specific temperature at a designated location. It serves as a reference temperature which gives relative distance of thermophysical properties relevant to the physical process. Using (14) and (15), (12) is expressed as:

$$
\frac{d}{d t} m_{d, i}=-\frac{V P_{i}\left(T_{a p p, d}\right) \times f_{d, i}}{\sum_{i} V P_{i}\left(T_{a p p, d}\right) \times f_{d, i}} \cdot \dot{m}_{E V, d} .
$$

Calculation of diffusion coefficient, $D$, for multi-component fuel is approximated here by mass-weighted average of diffusion coefficients of each fuel component and each coefficient may be looked up from [26] which gives functional expression in temperature. The following relation that kinetic theory would pose under ideal gas behavior assumption is also utilized [9], [10]:

$$
D \sim \frac{T_{a p p, d}^{3 / 2}}{p_{m}} .
$$

Calculation of normal vapor pressures, $V P_{i}$, can be also done by the functional expression in temperature provided in [26].

Let $X_{i}$ denote the ratio of the wall-impacting mass of $i$ th component to the total injected mass of fuel, which is the output of the droplet evaporation model and is the input to the single puddle vaporization model which will be discussed in the following section. The mass balance is then expressed as:

$$
f_{i}-X_{i}=\frac{1}{m_{d}^{0}} \int_{0}^{t_{e v}} \dot{m}_{E V, d, i} \cdot e^{-\frac{t}{\tau_{D A}}} d t .
$$

where $\tau_{D A}$ is the time constant of the decay of the number of airborne droplets, $t_{e v}$ is the time for complete vaporization of single droplet, i.e. $\left.m_{d}\right|_{t=t_{e v}}=0$. The time constant $\tau_{D A}$ is a universal tuning constant over the whole range of ethanol content, which should be significantly smaller than one engine cycle duration.

According to the model from (11)-(21), $X_{i}$ is varying in accordance with the variation of engine coolant temperature, $E C T$, manifold absolute pressure, $p_{m}$, and also with the variation of the ethanol content, $e$, i.e., $X_{i}=X_{i}\left(e, E C T, p_{m}\right)$. If we consider the effect of flow of air, then Reynolds number and Schmidt number should be involved in the formulation; in this case, $X_{i}$ may be also dependent on the air charge mass, engine RPM, and the port diameter as in [18] or [13]. Nevertheless, the stagnant air assumption may well capture the whole process of droplet evaporation or the spray injection process in port fuel injection (PFI) engines considering the usual timing of the fuel injection to the intake port before the intake valves open. Fig. 4 shows the wall-impacting fraction for the four gasoline components $X_{i}, i=1, \cdots, 4$ for E0 and E43, and ethanol $X_{5}$ for E100 and E43 as an example of the wall impacting fractions after the model calibration and the fuel simplification discussed in Section VI. The last subplot of Fig. 4 shows the wall-impacting fraction for ethanol. Fig. 4 depicts that lighter fuel components have relatively small $X_{i}$ among gasoline components since more droplet mass is evaporated before hitting the port wall, e.g., cyclo-hexane has very small number of $X_{i}$ even though its nominal mass fraction in gasoline is the highest, i.e., 0.65 in Table I.

The evolution of the droplet evaporation and the resulting wall impacting mass fractions can be computed off-line and 

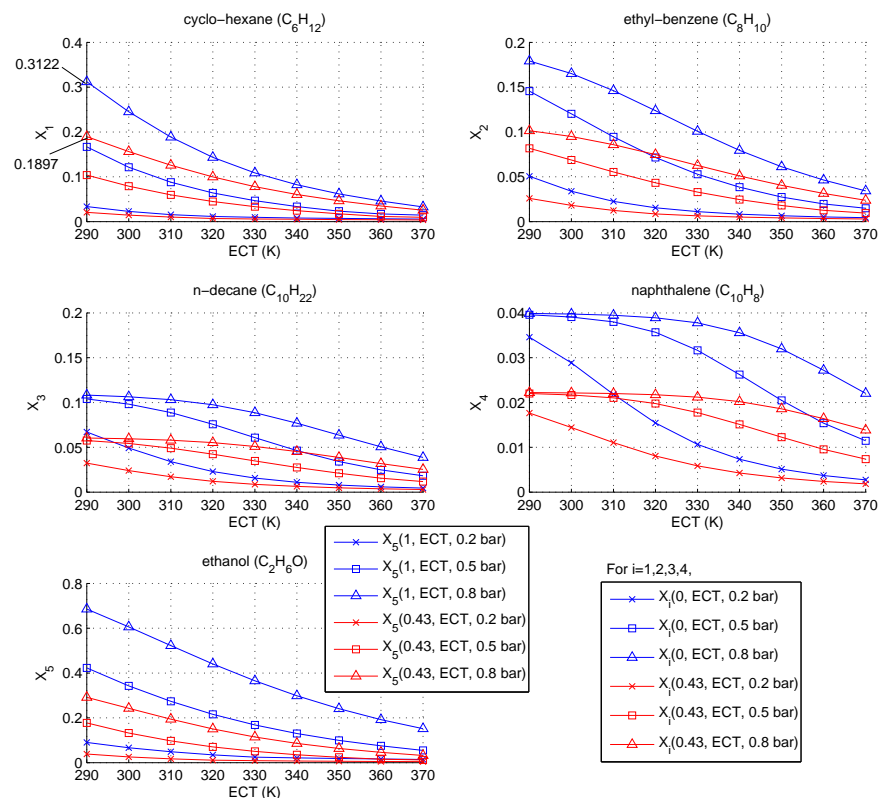

Fig. 4. Wall-impacting factor of gasoline fuel component $\left(X_{i}\left(0, E C T, p_{m}\right)\right.$ and $\left.X_{i}\left(0.43, E C T, p_{m}\right) i=1, \cdots, 4\right)$ and ethanol $\left(X_{5}\left(1, E C T, p_{m}\right)\right.$ and $\left.X_{5}\left(0.43, E C T, p_{m}\right)\right)$ at $p_{m}=0.2,0.5,0.8 \mathrm{bar}$

produce a five (5) three-dimensional $\left(e, E C T, p_{m}\right)$ look-up table which can be used in the fuel puddle evaporation process. By observing the trends of the computed $X_{i}$ for the different blends (after the tuning and the fuel composition reduction discussed later in Section VI) in Fig. 4, one can see that for example, cyclo-hexane shows that at $E C T=290 \mathrm{~K}$ and $p_{m}=0.8$ bar the computed impact factor for E43 is $0.1897=$ $X_{1}(0.43,290,0.8) \approx(1-0.43) X_{1}(0,290,0.8)=0.1780$. This motivated the computation of

$$
\begin{aligned}
\hat{e}_{i} & =1-\frac{X_{i}\left(e, E C T, p_{m}\right)}{X_{i}\left(0, E C T, p_{m}\right)} \text { for } i=1, \cdots, 4, \\
\hat{e}_{5} & =\frac{X_{5}\left(e, E C T, p_{m}\right)}{X_{5}\left(1, E C T, p_{m}\right)} .
\end{aligned}
$$

shown in bars in Fig. 5. Fig. 5 shows that the computed values of $\hat{e}_{i}$ were approximately equal to the fuel ethanol content, $\hat{e}_{i} \approx e$ for all $i=1, \cdots, 5$ leading to linear interpolation with respect to ethanol concentration for the impacting factor of each fuel component without loss of accuracy. The multicomponent fuel wall impacting factors can then be stored as five (5) two-dimensional $\left(E C T, p_{m}\right)$ look-up tables:

$$
\begin{aligned}
& X_{i}\left(e, E C T, p_{m}\right)=(1-e) X_{i}\left(0, E C T, p_{m}\right) \\
& \text { for } i=1, \cdots, 4, \\
& X_{5}\left(e, E C T, p_{m}\right)=e X_{5}\left(1, E C T, p_{m}\right) .
\end{aligned}
$$

Specifically, Fig. 5 shows the computed $\hat{e}_{i}$ for different gasoline-ethanol mixtures $(22 \%, 45 \%, 64 \%$, and $85 \%)$ in bars of blue, cyan, yellow, and red colors and at three different coolant temperatures $(E C T)$ and two manifold pressures. Fig. 5 suggests that the linear interpolation, (22) and (23), is especially a good approximation in low engine coolant temperature range, where the wall-impacting mass fraction is relatively high as shown in Fig. 4 and hence more accuracy

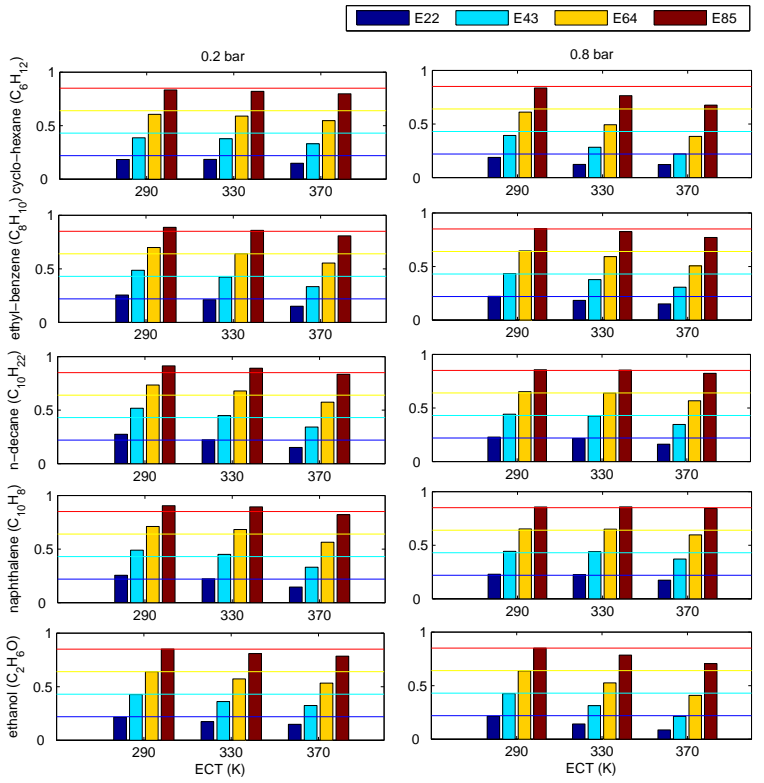

Fig. 5. Effective ethanol fraction $\hat{e}_{i}$ in linear interpolation of $X_{i}$ for each fuel component

is necessary. For more accuracy, the 5 wall impacting factors $X_{i}\left(e, E C T, p_{m}\right)$ could be stored as three dimensional maps as a function of finer fuel volumetric ethanol content than the one suggested from (22)-(23) based on the two end compositions, namely E0 and E100.

Note that the total wall-impacting mass fraction of the injected fuel is the sum of each $X_{i}$ over all fuel components, $X=\sum_{i} X_{i}$. The total wall-impacting mass fraction, $X$, may be compared with certain reference values, e.g., $X$ obtained by the least squares fit from section II, for initial rough calibration. However, $X$ alone cannot capture the vaporization process of fuel puddle and both of the wall-impacting mass fraction of each fuel component, $X_{i}$, and aggregate factor $X$ are finally implemented in the single puddle vaporization model which will be discussed in the next section.

\section{Single Puddle Vaporization Model}

Some droplets of the injected fuel hit the port wall before their complete evaporation. That mass forms a fuel puddle on the port wall and some portion of the puddle also vaporizes from the puddle. The mass of each component in the fuel puddle at intake valve opening (IVO) is equal to the sum of the previous-cycle mass and the portion of the newly injected fuel that hits the puddle. The total puddle mass at IVO is equal to the sum of masses of each component:

$$
\begin{aligned}
m_{p, i}^{I V O}(k)= & m_{p, i}(k-1)+X_{i}\left(e, E C T, p_{m}\right) \times m_{i n j}(k), \\
& i=1, \cdots, j, \\
m_{p}^{I V O}(k)= & \sum_{i=1}^{j} m_{p, i}^{I V O}(k),
\end{aligned}
$$

where $k$ denotes the event or cycle number, $i$ is index for fuel component and $j$ is the total number of fuel components $(j=5)$. It is assumed that the rest of injected fuel is evaporated 
and enter the cylinder on the intake stroke. The puddle mass at intake valve closing (IVC), $m_{p}$, is derived from Locatelli's model [13]. Assuming a cylindrical fuel puddle along the port wall, let $\delta_{t h}$ be the thickness of the puddle which is assumed constant along the height of the fuel puddle. The height of the puddle is varying according to the mass variation of the puddle. The vaporization rate is expressed as:

$$
\begin{aligned}
\dot{m}_{E V, p} & =\frac{\rho \cdot A_{p}}{d_{i n}} \cdot D_{p} \cdot S h_{p} \cdot \ln \left(1+B_{p}\right), \\
& =\frac{\breve{m}_{p}}{d_{i n} \cdot \delta_{t h}} \cdot D_{p} \cdot S h_{p} \cdot \ln \left(1+B_{p}\right),
\end{aligned}
$$

where $\rho$ is the density of the fuel puddle, $A_{p}$ is the surface area of the puddle exposed to air flow, $d_{i n}$ is the port diameter, $D_{p}$ is the diffusion coefficient, $S h_{p}$ is the Sherwood number, $B_{p}$ is the transfer number and $\breve{m}_{p}$ is the instantaneous puddle mass such that $\dot{m}_{E V, p}=-\dot{\check{m}}_{p}$. The fuel puddle mass at IVC, $m_{p}(k)$, is then obtained by integrating (27), for one cycle duration:

$$
m_{p}(k)=m_{p}^{I V O}(k)\left(1+B_{p}\right)^{-\alpha}
$$

where

$$
\alpha \triangleq C(e) \cdot \frac{120}{N} \cdot \frac{D_{p} \cdot S h_{p}}{d_{i n} \cdot \delta_{t h}} .
$$

In the above equation, $N$ denotes the engine RPM and a correction factor, $C$, parameterized by the ethanol content, $e$, is introduced to correct the effect of variation of fuel film thickness according to the ethanol content variation. The correction factor is modeled as a linear variation with the change of the ethanol content:

$$
C(e)=1-\sigma e,
$$

where $\sigma$ is a tuning constant. The evaporated mass is expressed as:

$$
\begin{aligned}
m_{\text {evap }}(k) & \triangleq m_{p}^{I V O}(k)-m_{p}(k) \\
& =m_{p}^{I V O}(k)\left[1-\left(1+B_{p}\right)^{-\alpha}\right] \\
& =\bar{\alpha} \cdot m_{p}^{I V O}(k),
\end{aligned}
$$

where the mass fraction of evaporation from the mass at IVO is defined as $\bar{\alpha} \triangleq 1-\left(1+B_{p}\right)^{-\alpha}$. Sherwood number is computed as follows [24]:

$$
\begin{aligned}
u_{A \infty} & =\frac{m_{a i r} \cdot N}{120} \cdot \frac{4}{\rho_{A} \cdot \pi \cdot d_{i n}^{2}}, \\
R e_{p} & =\frac{u_{A \infty} \cdot d_{i n}}{\nu_{A}}, \\
S c_{p} & =\frac{\nu_{A}}{D_{p}}, \\
S h_{p} & =0.023 \cdot R e_{p}^{0.83} S c_{p}^{0.44},
\end{aligned}
$$

where $\rho_{A}$ denotes density of air, $\nu_{A}$ denotes kinematic viscosity of air, and $m_{\text {air }}$ denotes air charge mass per cycle. Let us define the mass fraction of each component in the puddle at IVO:

$$
f_{p, i}(k) \triangleq \frac{m_{p, i}^{I V O}(k)}{m_{p}^{I V O}(k)} .
$$

Let $V P_{i}$ denote the normal vapor pressure of each fuel component as used in the droplet evaporation model. The diffusion coefficient, $D_{p}$, and the normal vapor pressures, $V P_{i}$ are evaluated at another apparent temperature for the fuel puddle vaporization:

$$
T_{a p p, p}=E C T-e \cdot \eta_{p} \cdot\left(E C T-E C T^{0}\right),
$$

where $\eta_{p}$ is another tuning constant and $E C T^{0}$ is the same tuning constant used in the droplet evaporation model. Calculation of $D_{p}$ is similarly performed to the droplet evaporation model (20) as $D_{p} \sim T_{a p p, p}^{3 / 2} / p_{m}$. The variable $B_{p}$ is computed similar to the droplet evaporation model as follows:

$$
\begin{aligned}
P P_{a i r}(k) & =p_{m}(k)-\frac{\sum_{i} V P_{i} \times f_{p, i}(k) / M_{i}}{\sum_{i} f_{p, i}(k) / M_{i}}, \\
B_{p}(k) & =\frac{\sum_{i} V P_{i} \times f_{p, i}(k)}{P P_{a i r}(k) \times M_{a i r} \times \sum_{i} f_{p, i}(k) / M_{i}} .
\end{aligned}
$$

The computation at the step $k$ is completed by updating the masses of each fuel component at the end of the intake stroke accounting for the evaporated fuel:

$$
\begin{aligned}
m_{\text {evap }, i}(k)= & \min \left\{m_{p, i}^{I V O}(k), m_{\text {evap }}(k) \times\right. \\
& \left.\frac{V P_{i}\left(T_{a p p, p}\right) \times m_{p, i}^{I V O}(k)}{\sum_{i} V P_{i}\left(T_{a p p, p}\right) \times m_{p, i}^{I V O}(k)}\right\}, \\
m_{p, i}(k)= & m_{p, i}^{I V O}(k)-m_{\text {evap }, i}(k) .
\end{aligned}
$$

Finally, the mass of fuel inducted into the cylinder is:

$$
\begin{aligned}
m_{f, c y l}(k)= & \left(1-X\left(e, E C T, p_{m}\right)\right) \times m_{i n j}(k) \\
& +\sum_{i=1}^{j} m_{\text {evap }, i}(k) .
\end{aligned}
$$

We can compute air-to-fuel ratio, $A F R$ and $\lambda$ at each step as follows:

$$
\begin{aligned}
A F R(k) & =\frac{m_{a i r}(k)}{m_{f, c y l}(k)}, \\
\lambda(k) & =\frac{A F R(k)}{A F R_{s}(k)},
\end{aligned}
$$

where the stoichiometric air-to-fuel ratio is computed by:

$$
A F R_{s}=9 \times e_{m}+14.6 \times\left(1-e_{m}\right) .
$$

Fig. 3 depicts the model summary where inputs to each submodel and tuning constants associated with each submodel are shown. In the droplet evaporation model, the parameter $d_{d}^{0}$ indicates the initial droplet diameter right after injection. The droplet evaporation model passes $X_{i}$ over to the singlepuddle vaporization model as inputs. Since $X_{i}$ is an integration result according to (21), the internal states during the droplet evaporation simulation for implementation at every numerical computation time step do not have to be known. Therefore, to avoid unnecessary increase of on-line computation time, which might be caused by computing $X_{i}$ at every engine cycle, the droplet evaporation model is simulated off-line to generate $X_{i}$ and the results are implemented as engine maps in the single-puddle vaporization model which updates its states every engine cycle. 


\section{Vi. Simulation}

The resulting model takes 6 inputs, $e, E C T, p_{m}, m_{a i r}$, $m_{i n j}, R P M$, and uses 8 parameters, $\tau_{D A}, d_{d}^{0}, \gamma, \eta_{d}, E C T^{0}$, $\delta_{t h}, \sigma, \eta_{p}$ to produce the fuel amount inducted into the cylinder and $\lambda$ as shown in Fig. 3. The effect and topology of the 8 parameters in the model structure is physically motivated. The droplet evaporation model depends on two parameters: the droplet decay time constant $\tau_{D A}$ introduced in the model in [13] and the droplet diameter $d_{d}^{0}$ which is physically necessary and is tuned according to the $\tau_{D A}$ setting. The puddle vaporization model depends on two physically motivated parameters: the puddle thickness $\delta_{t h}$ and $\sigma$ introduced to consider actual variation of $\delta_{t h}$ on ethanol content. The vaporization process depends on, and at the same time, influences temperature through 4 parameters, $\gamma, \eta_{d}, \eta_{p}$ and $E C T^{0}$. These 4 parameters (two for the droplet and two for the film) are used to account for the vaporization effect on surface temperature approximated as apparent temperature. This number of parameters in the ethanol-temperature relationship is not actually too high if one considers using an affine fit for each fuel blend we used for calibration. For parameter tuning and the model validation, the three different experiments of [2] have been obtained.

The experimental data show the $\lambda$ traces measured by a Universal Exhaust Gas Oxygen (UEGO) sensor for different fuel blends of E0, E22 and E85, respectively, while the 4.6L $2 \mathrm{~V}$ engine operates from cold start till full warm-up for each blend and several steps of tip-ins and tip-outs are applied.

As stated in section III, the multi-component model fuel for gasoline was first taken from the mass fractions in [5]. The 8 parameters associated with our model were then tuned for that model fuel which uses 8 chemical compounds including ethanol. The tuning of the model parameters is explained below. First, the initial droplet diameter, $d_{d}^{0}$, was chosen and the time constant, $\tau_{D A}$, was tuned so that the whole droplet evaporation process around the engine coolant temperature range $(290-370 \mathrm{~K})$ and the manifold absolute pressure range (0.1-0.8 bar) yielded a reasonable range of the wall impacting factors, $X_{i}$. The value for $E C T^{0}$ was determined to be close to ambient temperature. The product of $\gamma$ and $\eta_{d}, \gamma \cdot \eta_{d}$ was tuned to yield proper shapes of $\lambda$ excursions for E0, and then each parameter was determined by matching the $\lambda$ excursions for E85. Likewise, the film thickness, $\delta_{t h}$, was tuned to match the film vaporization rate for $\mathrm{E} 0 ; \sigma$ and $\eta_{p}$ were tuned to match the data for E85. In this way, true validation is obtained through the comparison between simulation and experiments for E22.

Table II summarizes the tuned parameters and their values. The port diameter, $d_{i n}$ is $0.045 \mathrm{~m}$. Using these parameters, the gasoline fuel components were then reduced to yield similar model simulation results to those of the previously used gasoline fuel components of [5]. Table I summarizes the optimized fuel fractions in the last column and the following simulations used those fuel fractions associated with a 4component gasoline instead of the original 7 components in [5]. As previously discussed in section IV, $X_{i}$ for gasoline components $(i=1, \cdots, 4)$ and $X_{5}$ for ethanol are calibrated
TABLE II

TUNED PARAMETERS OF SIMULATION

\begin{tabular}{|c|c|c|c|}
\hline Parameter & Value & Unit & Model \\
\hline$\tau_{D A}$ & $8.0 \times 10^{-5}$ & {$[\mathrm{~s}]$} & \multirow{4}{*}{ Droplet evaporation } \\
\hline$d_{d}^{0}$ & $6.0 \times 10^{-5}$ & {$[\mathrm{~m}]$} & \\
\hline$\gamma$ & 0.80 & - & \\
\hline$\eta_{d}$ & 0.31 & - & \\
\hline$E C T^{0}$ & 290 & {$[\mathrm{~K}]$} & $\begin{array}{l}\text { Droplet evaporation and } \\
\text { single-puddle vaporization }\end{array}$ \\
\hline$\sigma$ & 0.60 & - & \multirow{3}{*}{ Single-puddle vaporization } \\
\hline$\eta_{p}$ & 0.75 & - & \\
\hline$\delta_{t h}$ & $3.0 \times 10^{-3}$ & {$[\mathrm{~m}]$} & \\
\hline
\end{tabular}

for E0 and E100 cases, respectively, and all the intermediate $X_{i}$ 's for intermediate fuel blends are interpolated by (22) and $(23)^{3}$. Further work is needed for establishing a further simplification an or automated parameter calibration technique. A detailed analysis of the model sensitivity on small fuel variability, such as summer and winter grade gasoline, is also required in the future.

Fig. 6 shows the inputs to the (puddle vaporization) model for E0, based on the warm-up tests performed in [2]. Similar inputs obtained from the tests performed in [2] were used for E22 and E85, although not shown here. The air charge associated with the six inputs was estimated using the methodology of [8] and [22] and Ford proprietary calibration of the manifold filling dynamic model and the cylinder pumping.

Fig. 7 shows the simulated fuel puddle mass and ethanol mass fraction in the puddle for each fuel case. The lighter fuel components would vaporize faster than the heavier, so the puddle mass and composition would evolve in time. Fig. 7 shows that the model predicts different fuel puddle composition from the injected fuel. Fig. 8-Fig. 10 show the simulated $\lambda$ responses using the proposed model for E0, E22 and E85, respectively, compared with the experimental data measured by a UEGO sensor. The agreement between the simulations and the experimental data is quite reasonable. Model matching is especially very good for sufficiently warmed-up operations. The model also predicts the overall decrease in magnitude of the $\lambda$ excursions well. It also captures the asymmetric excursions due to tip-ins and tip-outs observed in the experimental data. The verification needs to be confirmed at more operating points to evaluate the model accuracy. However, the provided simulation and experimental results reasonably support the validity of the proposed model for at least some operating conditions.

\section{TRANSIENT FUEL COMPENSATOR}

Transient fuel compensation is realized by inverting the dynamics of the fuel film and injection. This means, for a linear fuel puddle model, cancelation of pole and zero in fuel puddle dynamics with zero and pole in TFC transfer function [1], [15]. However, direct inversion of pole-zero cancelation as

\footnotetext{
${ }^{3}$ Calibration for $X_{5}$ with respect to E85 data is first performed and $X_{5}$ for E100 is then obtained using the calibrated parameters.
} 

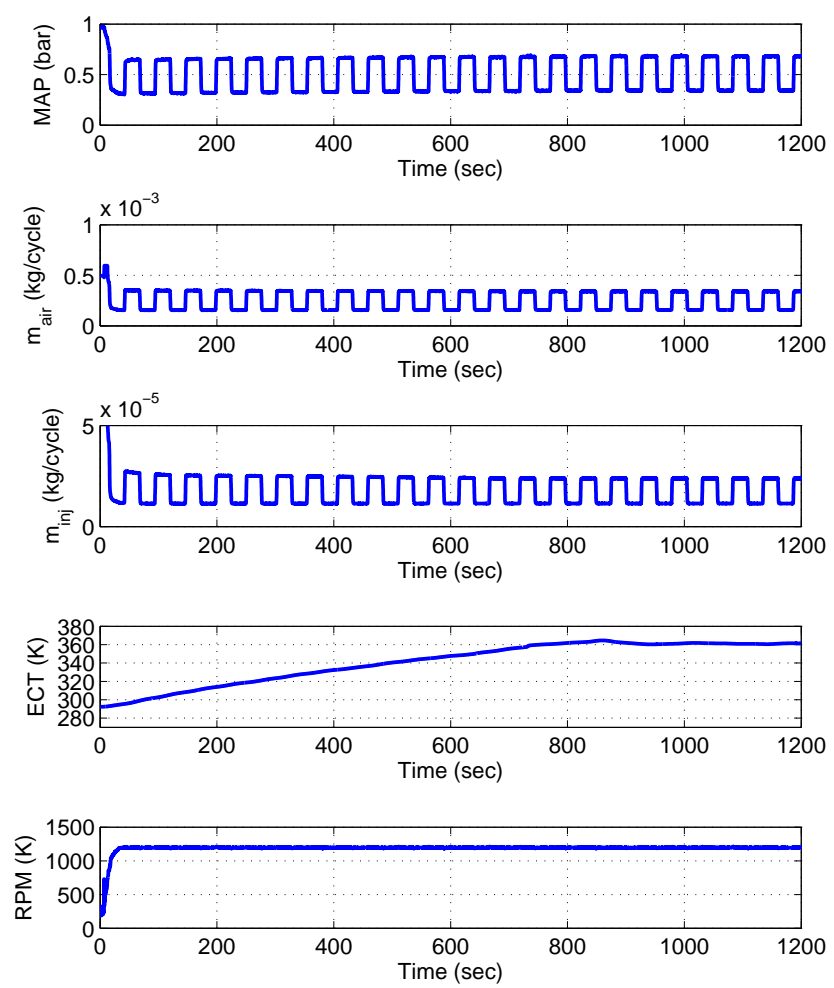

Fig. 6. Fuel injected and other measurements from [2] used for the model inputs (E0)
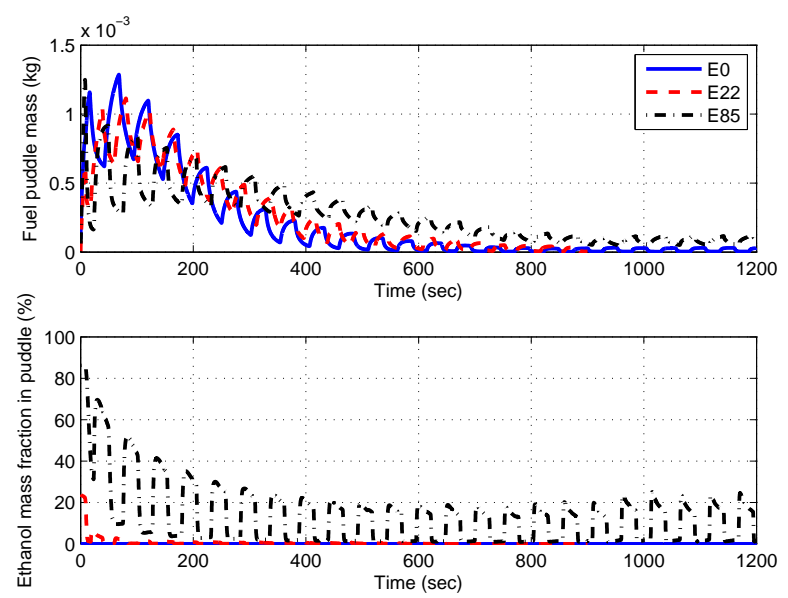

Fig. 7. Simulated puddle mass and ethanol mass fraction in puddle for each fuel

in a linear model is not available for our fuel puddle model. To design a transient fuel compensator, the current fuel injection, $m_{i n j}(k)$, should be expressed as a function of previous states, current output and other current inputs since current states are not available. To this end, (39) can be utilized. However, the term $\sum_{i=1}^{j} m_{\text {evap }, i}(k)$ is expressed involving complicated nonlinearity in $m_{i n j}(k)$, hence causing difficulty in getting the inverted expression of $m_{i n j}(k)$ given a desired fuel in the cylinder, $m_{f, c y l}^{\text {des }}(k)$. A reasonable approximation can be utilized to remedy this problem. First, let us express $m_{\text {evap }, i}(k)$
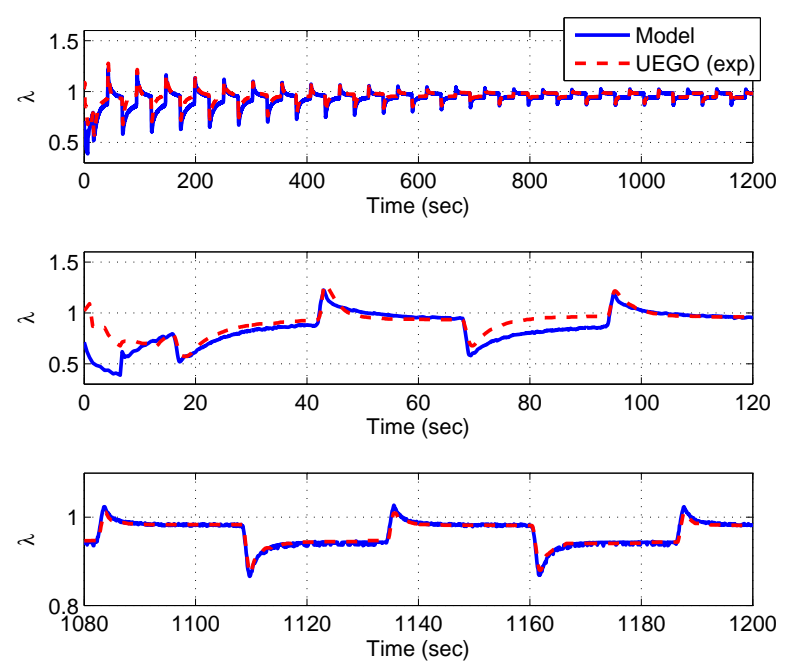

Fig. 8. Simulated and experimental $\lambda$ response for E0
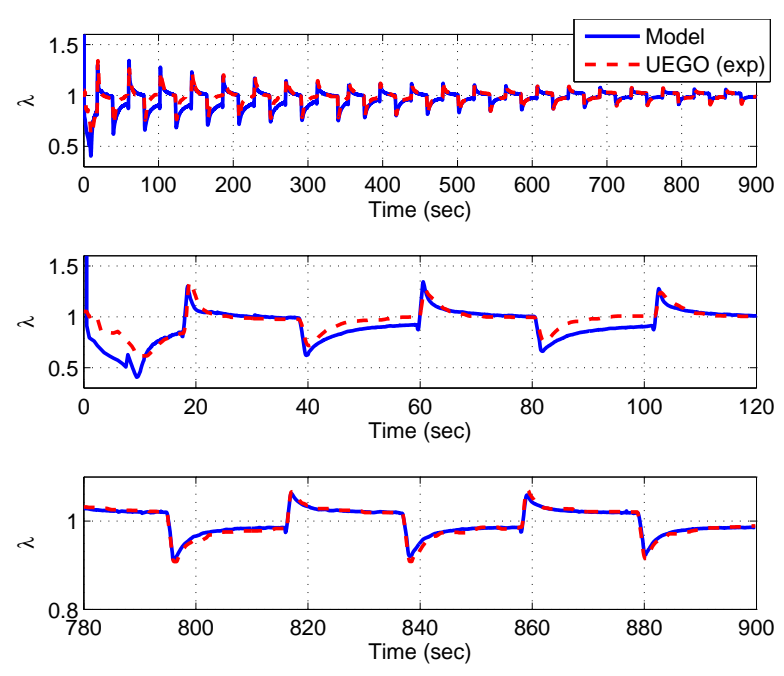

Fig. 9. Simulated and experimental $\lambda$ response for E22
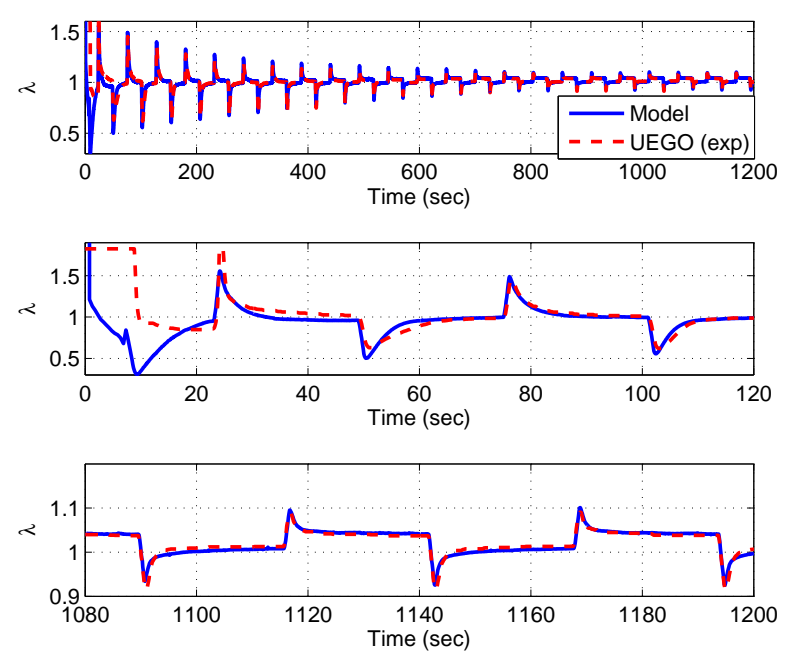

Fig. 10. Simulated and experimental $\lambda$ response for E85 
as follows using (37) and (31):

$$
m_{\text {evap }, i}(k)=g_{i}(k) \cdot m_{p, i}^{I V O}(k),
$$

where

$$
g_{i}(k) \triangleq \min \left\{1, \bar{\alpha}(k) \frac{V P_{i}(k) \times m_{p}^{I V O}(k)}{\sum_{i} V P_{i}(k) \times m_{p, i}^{I V O}(k)}\right\} .
$$

The normal vapor pressure of $i$-th component at the current step $k, V P_{i}(k)$, can be calculated using the current engine coolant temperature, $\operatorname{ECT}(k)$, and the current ethanol content, $e(k)$. Using (43) and (24), (39) is then expressed as:

$$
\begin{aligned}
m_{f, c y l}(k)= & (1-X(k)) \cdot m_{i n j}(k)+\sum_{i=1}^{j} g_{i}(k) \cdot m_{p, i}^{I V O}(k) \\
= & \left(1-X(k)+\sum_{i} g_{i}(k) \cdot X_{i}(k)\right) \cdot m_{i n j}(k) \\
& +\sum_{i} g_{i}(k) \cdot m_{p, i}(k-1),
\end{aligned}
$$

where the current total wall impacting fraction, $X(k)$, and the current wall impacting factor for each fuel component, $X_{i}(k)$, are obtained using the current ethanol content, $e(k)$, the current engine coolant temperature, $\operatorname{ECT}(k)$, and the current manifold absolute pressure, $p_{m}(k)$. To eliminate dependency of $g_{i}(k)$ on $m_{i n j}(k)$ in (45), we use a simplifying assumption that the composition of the fuel puddle is not affected significantly by the difference between the current injection, $m_{i n j}(k)$, and the previous injection, $m_{i n j}(k-1)$. This assumption is reasonable for a large puddle mass, when higher accuracy of a transient fuel compensation is required. We can then approximate $g_{i}(k)$ in (44) as:

$$
\hat{g}_{i}(k)=\min \left\{1, \hat{\bar{\alpha}}(k) \frac{V P_{i}(k) \times \hat{m}_{p}^{I V O}(k)}{\sum_{i} V P_{i}(k) \times \hat{m}_{p, i}^{I V O}(k)}\right\},
$$

where

$$
\begin{aligned}
& \hat{m}_{p, i}^{I V O}(k)=m_{p, i}(k-1)+X_{i}(k) \times m_{i n j}(k-1), \\
& \hat{m}_{p}^{I V O}(k)=\sum_{i=1}^{j} \hat{m}_{p, i}^{I V O}(k) .
\end{aligned}
$$

and

$$
\hat{\bar{\alpha}}(k)=1-\left(1+\hat{B}_{p}(k)\right)^{-\alpha(k)} .
$$

In (49), $\alpha(k)$ can be calculated using the current inputs according to (29) and the approximation of the transfer number at the current step $k, \hat{B}_{p}(k)$, can be computed as:

$$
\hat{B}_{p}(k)=\frac{\sum_{i} V P_{i}(k) \times \hat{f}_{p, i}(k)}{\widehat{P P}_{a i r}(k) \times M_{a i r} \times \sum_{i} \hat{f}_{p, i}(k) / M_{i}},
$$

where

$$
\begin{aligned}
\hat{f}_{p, i}(k) & =\frac{\hat{m}_{p, i}^{I V O}(k)}{\hat{m}_{p}^{I V O}(k)} \\
\widehat{P P}_{a i r}(k) & =p_{m}(k)-\frac{\sum_{i} V P_{i}(k) \times \hat{f}_{p, i}(k) / M_{i}}{\sum_{i} \hat{f}_{p, i}(k) / M_{i}} .
\end{aligned}
$$

Using the approximation, (46), the transient fuel compensation is then calculated from (45) as:

$$
m_{i n j}^{T F C}(k)=\frac{m_{f, c y l}^{\text {des }}(k)-\sum_{i} \hat{g}_{i}(k) \cdot m_{p, i}(k-1)}{1-X(k)+\sum_{i} \hat{g}_{i}(k) \cdot X_{i}(k)},
$$

where

$$
m_{f, c y l}^{\text {des }}(k) \triangleq \frac{m_{a i r}(k)}{A F R_{s}(k)} .
$$

One easier way to approximate $g_{i}(k)$ is to use previously calculated $g_{i}$ in the puddle model so that $\hat{g}_{i}(k)=g_{i}(k-1)$ without recalculation of $\hat{g}_{i}(k)$ as above. This simpler approximation, however, results in poorer TFC performance as shown and discussed later in Fig. 12. Fig. 11 shows the $\lambda$ and the

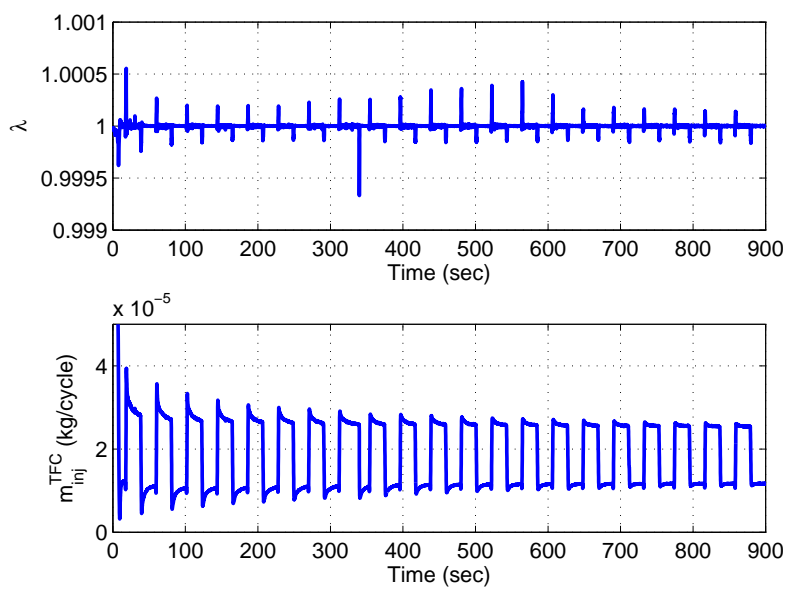

Fig. 11. Simulation of the transient fuel compensation for E22

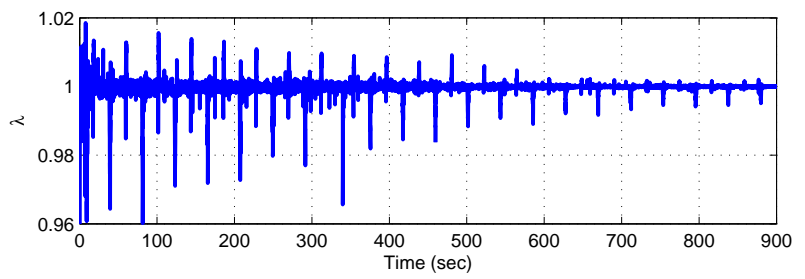

Fig. 12. Simulation of the transient fuel compensation for E22 with a simpler approximation, $\hat{g}_{i}(k)=g_{i}(k-1)$

injected fuel using the compensator in (53) to demonstrate the accuracy of our assumption and the approximation using (46)(52), while Fig. 12 shows the simulated $\lambda$ with the TFC based on a simpler (but cruder) approximation, $\hat{g}_{i}(k)=g_{i}(k-1)$. The simulation was done under the assumption that the plant follows the model perfectly and the states are known. A simulation using the model for E22 with inputs $p_{m}, m_{\text {air }}$, $E C T$ and $N$ as prescribed in the experimental data used in section VI, and $m_{i n j}$ as prescribed from the proposed TFC in (46)-(54) tuned for E22 is shown in Fig. 11. The simulated $\lambda$ trace in Fig. 11 shows several spikes due to the errors caused by the approximation but their amplitudes are negligible. This AFR accuracy is of course achieved only with very well tuned fuel puddle model parameters and known ethanol percentage. 


\section{CONCLUSION}

In this paper, a physics-based fuel puddle model for any ethanol-gasoline mixture is developed. This model may be used for air-to-fuel ratio control in FFVs with engines equipped with port fuel injection. First, a multi-component fuel comprised of five chemical compounds is parameterized by ethanol content and is utilized to characterize gasoline-ethanol blends. The model consists of a droplet evaporation model and single-puddle vaporization model. The droplet evaporation model is pre-simulated off-line to generate port wall-impacting factors of the injected multi-component fuel to be used in single-puddle. A single-puddle vaporization model is then utilized as a cycle-based model that may be simulated on-line to characterize the fuel puddle dynamics in port fuel injected engines. To verify the validity of the model, simulation results are compared with a limited number of experimental data. A transient fuel compensator using the proposed model is also formulated. Since direct pole-zero cancelation is not possible for the nonlinear model, an approximation is used to invert the model to design a TFC. The TFC approximation is demonstrated with a simulation.

Further work will be needed to reduce the model complexity and define the minimum set of tunable parameters. This future investigation could lead to on-board identification of the flexfuel puddle model.

\section{REFERENCES}

[1] C. F. Aquino, "Transient a/f control characteristics of the 5 liter central fuel injection engine," SAE paper 810494.

[2] J. J. Batteh and E. W. Curtis, "Modeling transient fuel effects with alternative fuels," SAE paper 2005-01-1127.

[3] J. J. Batteh, E. W. Curtis, and M. Fried, "Analytical assessment of simplified transient fuel tests for vehicle transient fuel compensation," SAE paper 2005-01-3894.

[4] E. W. Curtis, C. F. Aquino, D. K. Trumpy, and G. C. Davis, "A new port and cylinder wall wetting model to predict transient air/fuel excursions in a port fuel injected engines," SAE paper 961186.

[5] E. W. Curtis, S. Russ, C. Aquino, G. Lavoie, and N. Trigui, "The effects of injector targetting and fuel volatility on fuel dynamics in a PFI engine during warm-up," SAE paper 982519.

[6] R. C. Delgado, A. S. Araujo, and V. J. Fernandes Jr., "Properties of Brazilian gasoline mixed with hydrated ethanol for flex-fuel technology," Fuel Processing Technology, vol. 88, no. 4, pp. 365-368, 2007.

[7] M. L. Greenfield, G. A. Lavoie, C. S. Smith, and E. W. Curtis, "Macroscopic model of the D86 fuel volatility procedure," SAE paper 982724.

[8] J. Grizzle, J. Cook, and W. Milam, "Improved cylinder air charge estimation for transient air fuel ratio control," American Control Conference, 1994, vol. 2, pp. 1568-1573, 1994.

[9] L. Guzzella and C. H. Onder, Introduction to Modeling and Control of Internal Combustion Engine Systems. Springer, 2004.

[10] F. P. Incropera and D. P. DeWitt, Fundamentals of Heat and Mass Transfer, 5th ed. John Wiley \& Sons, 2002.

[11] M. Jankovic, S. Magner, D. Hagner, and Y. Wang, "Multi-input transient fuel control with auto-calibration," in Proceedings of the 2007 American Control Conference.

[12] K. Kar, T. Last, C. Haywood, and R. Raine, "Measurement of vapor pressures and enthalpies of vaporization of gasoline and ethanol blends and their effects on mixture preparation in an SI engines," SAE paper 2008-01-0317.

[13] M. Locatelli, C. H. Onder, and H. P. Geering, "An easily tunable wallwetting model for PFI engines," SAE paper 2004-01-1461.

[14] M. Locatelli, E. Alfieri, C. H. Onder, and H. P. Geering, "Identification of the relevant parameters of the wall-wetting system by extended Kalman filtering," Control Engineering Practice, vol. 14, no. 3, pp. 235-241, 2006.
[15] P. J. Maloney, "An event-based transient fuel compensator with physically based parameters," SAE paper 1999-01-0553.

[16] H. Melgaard, E. Hendricks, and H. Madsen, "Continuous identification of a four-stroke SI engine," in Proceedings of the 1990 American Control Conference, May 1990, pp. 1876-1881.

[17] K. Nakata, S. Utsumi, A. Ota, K. Kawatake, T. Kawai, and T. Tsunooka, "The effect of ethanol fuel on a spark ignition engine," SAE paper 200601-3380.

[18] J. Senda, T. Higaki, Y. Sagane, H. Fujimoto, Y. Takagi, and M. Adachi, "Modeling and measurement on evaporation process of multicomponent fuels," SAE paper 2000-01-0280.

[19] W. A. Sirignano, Fluid Dynamics and Transport of Droplets and Sprays. Cambridge University Press, 1999.

[20] J. S. Souder and J. K. Hedrick, "Adaptive sliding mode control of air-fuel ratio in internal combustion engines," International Journal of Robust and Nonlinear Control, vol. 14, no. 6, pp. 525-541, 2004.

[21] D. B. Spalding, Combustion and Mass Transfer. London: Pergamon Press, 1979.

[22] A. Stotsky and I. Kolmanovsky, "Application of input estimation techniques to charge estimation and control in automotive engines," Control Engineering Practice, vol. 10, no. 12, pp. 1371 - 1383, 2002.

[23] C. F. Taylor, Internal Combustion Engine in Theory and Practice. The MIT Press, 1985, vol. 2, revised edition.

[24] R. E. Treybal, Mass-Transfer Operations, 3rd ed. McGraw-Hill, 1980.

[25] G. F. Yao, S. I. Abdel-Khalik, and S. M. Ghiaasiaan, "An investigation of simple evaporation models used in spray simulations," Journal of Heat Transfer, vol. 125, no. 1, pp. 179-182, 2003.

[26] C. L. Yaws, Yaws' Handbook of Thermodynamic and Physical Properties of Chemical Compounds. Knover, 2003.

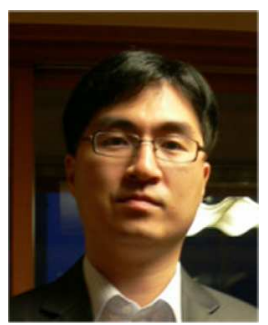

Kyung-ho Ahn received the B.S. and M.S. degrees in mechanical engineering from POSTECH, Pohang, South Korea, in 1999 and 2001, respectively. He is currently working towards the Ph.D. degree in mechanical engineering at the University of Michigan, Ann Arbor since 2006.

From 2001 to 2003, he worked at Korea Aerospace Industries, Ltd., as an engineer on the unmanned aerial vehicle (UAV) project. He also worked as a Senior Researcher at the Institute for Advanced Engineering on the grinding robot project from 2003 to 2005 in South Korea. Later, he has been a technical staff at the Korea Institute of Science and Technology on the humanoid robot project. His areas of current research interest include modeling, control and estimation in advanced powertrain systems.

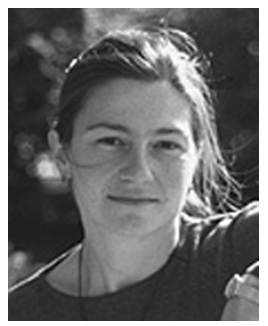

Anna G. Stefanopoulou obtained her Diploma (1991, Nat. Tech. Univ. of Athens, Greece) in Naval Architecture and Marine Engineering and her Ph.D. (1996, University of Michigan) in Electrical Engineering and Computer Science. She is a professor of mechanical engineering at the University of Michigan. She was an assistant professor (1998-2000) at the University of California, Santa Barbara, and a technical specialist (1996-1997) at Ford Motor Company. She is an ASME and an IEEE Fellow, an Associate Editor of the IEEE Transactions on Control System Technology and the ASME Journal of Dynamic Systems Measurements and Control. She has a book on Control of Fuel Cell Power Systems, nine US patents, 4 best paper awards and more than 120 publications. Her current work addresses the control and automation issues associated with fuel cells, fuel processing, and internal combustion engines. 


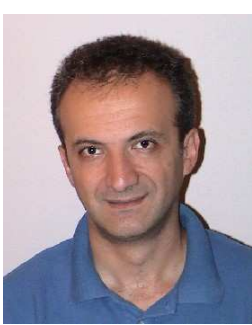

Mrdjan Jankovic (M'94-SM'99-F'04) received the B.S. (1986) degree from the University of Belgrade Yugoslavia, and the M.S. (1989) and Ph.D. (1992) degrees from Washington University in St. Louis. He held postdoctoral teaching and research positions with Washington University and UC Santa Barbara. He joined the Ford Research Laboratory, Dearborn, MI, in 1995 where he is currently a Technical Leader in the Powertrain Controls Department. Dr. Jankovic has coauthored one book, two book chapters, and more than 100 technical publications. $\mathrm{He}$ is a co- inventor on more than 30 U.S. patents, several of which are implemented in Ford products worldwide. His research interests include automotive engine control, nonlinear control, and time-delay systems. Dr. Jankovic received two Ford Research Technical Achievement Awards and best paper awards from IEEE, AVEC, and SAE for three automotive control papers. He is a past Associate Editor of the IEEE TRANSACTIONS ON CONTROL SYSTEMS TECHNOLOGY and past chair of several IEEE and SAE committees. 